J.-H. Kang • M.-J. Kim • S.-H. Ko • I.-K. Jeong •

K.-H. Koh · D.-J. Rhie · S.-H. Yoon ·

S.-J. Hahn • M.-S. Kim • Y.-H. Jo

\title{
Upregulation of rat $C$ cnd1 gene by exendin-4 in pancreatic beta cell line INS-1: interaction of early growth response-1 with cis-regulatory element
}

Received: 1 August 2005 / Accepted: 19 December 2005 / Published online: 18 March 2006

C) Springer-Verlag 2006

\begin{abstract}
Aims/hypothesis: The aim of this study was to investigate the effect of exendin- 4 on the expression of cyclin D1 gene (Ccndl), which is critical in regulating the progression of the cell cycle in INS-1 cells. Materials and methods: INS-1 cells were stimulated with exendin-4 $(10 \mathrm{nmol} / \mathrm{l})$. Transient transfection and luciferase reporter assays were performed to measure promoter activities of rat $C$ cndl. Electrophoretic mobility shift and chromatin immunoprecipitation assays were used to examine the binding of transcription factors to sites responsive to exendin- 4 in vitro and in vivo, respectively. Results: Exendin- 4 increased both $C c n d 1$ mRNA and its protein levels in a time-dependent manner. The region from -174 to +130 of the promoter was found to contain cis-regulatory elements responsible for exendin-4mediated gene induction. Early growth response-1 (EGR1) protein was bound to the region from -153 to -134 , which includes the putative EGR1 binding site (5'-CACCCCCGC-3'). Moreover, exendin-4 recruited
\end{abstract}

The first two authors contributed equally to this study.

J.-H. Kang · M.-J. Kim · D.-J. Rhie $\cdot$ S.-H. Yoon ·

S.-J. Hahn · M.-S. Kim · Y.-H. Jo ( $₫)$

Department of Physiology, College of Medicine,

Catholic University of Korea,

Seoul, South Korea

e-mail: yhjo@catholic.ac.kr

Tel.: +82-2-590-1170

Fax: $+82-2-532-9575$

S.-H. Ko

Department of Internal Medicine, College of Medicine, Catholic University of Korea,

Seoul, South Korea

I.-K. Jeong

Department of Internal Medicine, School of Medicine,

Hallym University,

Seoul, South Korea

K.-H. Koh

Department of Food Science and Nutrition,

Catholic University of Korea,

Puchon City, South Korea
EGR1 protein to the promoter in vivo. Conclusions/ interpretation: These findings suggest that exendin-4 activates Ccnd1 transcription through induction of EGR1 binding to a cis-regulatory element between -153 and -134 on the rat $C c n d 1$ promoter. These results provide an important indication that exendin-4 is a growth factor regulating beta cell proliferation.

Keywords CCND1 Cyclin D1 - Early growth response-1 $\cdot$ Exendin-4 $\cdot$ INS-1 cell

Abbreviations AP2: activating protein 2 - ATF: activating transcription factor - CCND1: cyclin D1 - ChIP: chromatin immunoprecipitation (assay) - CREB: cyclic AMP-responsive element binding protein - EGR1: early growth response 1 protein - GLP-1: glucagon-like peptide1 IEGs: immediate early genes - SP1: specific protein 1 . STAT5: signal transducer and activator transcription 5

\section{Introduction}

Glucagon-like peptide-1 (GLP-1) is a potent glucoincretin hormone secreted from the intestinal $\mathrm{L}$ cells $[1,2]$. It displays pleiotropic effects including beta cell proliferation and insulinotropic action [3-6]. Several immediate early genes (IEGs), which are implicated in the regulation of cell replication, are induced by GLP-1 [7]. The IEGs include the transcription factors c-fos, c-Jun, Jun B and Zif268. In pancreatic beta cells, the activator protein-1 (AP1) transcription factor consists of c-fos and c-Jun and is involved in the mitogenic signal for insulin receptor substrate [8]. Zif268, also known as EGR1, has been suggested to mediate beta cell growth as well as adaptive responses to sustained glucose stimulation [9]. Moreover, GLP-1 activates PI3-K and extracellular regulated kinase to induce target genes such as Fos (c-fos) Jun (c-Jun) and Egrl (zif268), leading to beta cell proliferation $[6,10]$. Thus, it is conceivable that GLP-1 might activate the cell cycles through induction of these transcription factors for proliferation of beta cells. 
The mammalian cell cycle is mainly regulated by cyclins that are associated with cyclin-dependent kinases [11, 12]. In particular, cyclin D1 (CCND1) plays a key role in progression of the cell cycle from the G1 to the S phase. Ccnd 1 expression appears to be predominantly regulated at the transcriptional level, although post-transcriptional mechanisms are also involved [13, 14]. The promoter region of Ccndl contains multiple potential cis-regulatory elements, including binding sites for AP1, signal transducer and activator transcription 5 (STAT5), EGR1, specific protein 1 (SP1) and activating transcription factor (ATF)/cyclic AMP-responsive element binding protein (CREB), which are important for the transcriptional activation of Ccnd1 [15-17]. The AP1 binding site has been shown to be involved in angiotensin II-induced activation of the $C c n d 1$ promoter in human adrenal cells [15]. The STAT5 binding site has been implicated in cytokine-dependent proliferation of haematopoietic cells [16]. Oestrogen-induced activation of Ccndl was shown to be mediated by the ATF/CREB binding site, in which ATF2 and c-Jun formed heterodimers [17]. These studies suggest that the transcriptional regulation of Ccndl may occur in a cell- and stimulus-specific manner. However, little is known about the transcriptional regulation of Ccndl in pancreatic beta cells in response to GLP-1.

We examined the effect of exendin-4, a potent GLP-1 agonist, using INS-1 cells as a model for pancreatic beta cells, on the expression of $C c n d 1$, and on the cis-regulatory regions and the trans-acting factors involved in the transcriptional activation of the gene.

\section{Materials and methods}

\section{Materials}

Cell culture media, exendin- 4 , poly $(\mathrm{dI}-\mathrm{dC})$ and cycloheximide were purchased from Sigma-Aldrich (St Louis, MO, USA). pGL3 basic, pSV40- $\beta$-galactosidase control vector and luciferase assay reagent were from Promega (Madison, WI, USA). pCR3.1 vector and Lipofectamine-Plus reagent were from Invitrogen (Carlsbad, CA, USA). Anti-mouse CCND1 (556470) was from Cell Signaling (Beverly, MA, USA). Polyclonal anti-SP1 (1C6), anti-EGR1 (C-19), antiAP2 (C-18), anti-tubulin (T 4026) antibodies, and EGR1, SP1 and AP2 consensus sequences were from Santa Cruz Biotechnology (Santa Cruz, CA, USA). The Quikchange Site-Directed Mutagenesis Kit was from Stratagene (La Jolla, CA, USA).

Isolation of pancreatic islets and culture of INS-1 cells

Primary pancreatic islets were isolated from SpragueDawley rats (Daehan Biolink Company, Chungbuk, Korea) using collagenase digestion and Ficoll gradient separation, as described previously [18]. INS-1 cells (between passages 10 and 19) were cultured in RPMI 1640 medium supplemented with $5.6 \mathrm{mmol} / \mathrm{l}$ D-glucose, 10\% FBS,
$2 \mathrm{mmol} / \mathrm{l}$ L-glutamine, $50 \mu \mathrm{mol} / \mathrm{l} \quad$ 2-mercaptoethanol, $1 \mathrm{mmol} / \mathrm{l}$ pyruvate, streptomycin $(100 \mathrm{U} / \mathrm{ml})$, and penicillin $(100 \mathrm{U} / \mathrm{ml})$ at $37^{\circ} \mathrm{C}$ under a $5 \% \mathrm{CO}_{2}$ atmosphere.

Western blot analysis

Western blot analysis was done as described previously [18]. Briefly, $30 \mu \mathrm{g}$ of total protein was separated on a $10 \%$ SDS-polyacrylamide gel. Following electrophoresis, proteins were transferred to nitrocellulose membrane (Schleicher \& Schuell, Keene, NH, USA). The membrane was blocked. The blots were then immunoblotted with antiCCND1, anti-tubulin or anti-EGR1 antibodies. Following incubation with the secondary antibodies, the membrane was visualised with a chemiluminescence system (Amersham Biosciences, Piscataway, NJ, USA).

Northern blot analysis

Northern blot analysis was performed as described previously [18]. Briefly, total RNA was extracted using STAT60 Reagent (TelTest, Friendswood, TX, USA) according to the manufacturer's instructions. Samples $(15 \mu \mathrm{g})$ were fractionated in a $1 \%$ formaldehyde agarose gel and transferred onto a nylon membrane (Schleicher \& Schuell). Hybridisation was performed in a rapid hybridisation buffer (Amersham biosciences) with a rat Ccndl riboprobe labelled with $\left[{ }^{32} \mathrm{P}\right]-\mathrm{dCTP}$ (specific activity 111 $\mathrm{TBq} / \mathrm{mmol}$; PerkinElmer Life Science, Boston, MA, USA). The blot was washed under high-stringency conditions and was subjected to autoradiography.

\section{Construction of serial-deletion rat $\operatorname{Ccnd1}$ promoters}

A 827-bp fragment of rat Ccnd1 promoter was amplified by PCR using $1 \mu \mathrm{g}$ of the genomic DNA from INS-1 cells. The PCR primers used were as follows: sense primer with the KpnI site (underlined), 5'-TGGGTACC GCCAGGATAAACCGGTCACTGTAAGAAG-3', antisense primer with XhoI (underlined), 5'-GGTAAGCTT GTGGGCTGTGGTCCGGGTTGGGTC-3'. The amplified product was cloned into pGL3-basic vector (pCD1827). To prepare serial deletion constructs, PCR was performed using pCD1-827 construct as a template. The sense primers with $K p n I$ sites were as follows: pCD1-625, sense primer (5'-TGGGTACCCTTTGCAACCACCC CAGTGC-3'); pCD1-446, sense primer (5'-TGGGTACC GAAACGCGCCCATTCTCTCC-3'); pCD1-174, sense primer (5'-TGGGTACCGGCAGGCTTTGGGTTTGTCC3'); pCD1-73, sense primer (5'-TGGGTACCGGCTTT GATCTCTGCTTAACAAC-3'); pCD1-34, sense primer (5'-TGGGTACCCTACGGAGTTTTGTTGAAGTTG-3'). The antisense primer was the same as that used for the construction of the pCD1-827. 
Site-directed mutagenesis

The pCD1mEgr-827 reporter plasmid was prepared using the QuikChange Site-Directed Mutagenesis Kit according to the manufacturer's protocol. The top strand of the primer set was as follows: 5'-GTTTGTCCCCCCCCACCC TTGCCGCTCACTGCTC-3'. The two underlined nucleotides in the high $\mathrm{GC}$ box were changed from $\mathrm{C}$ (wild type) to $\mathrm{T}$. The mutation was verified by DNA sequence analysis.

Transient transfection and luciferase activity assays

Constructs of the rat Ccnd1 promoter $(1 \mu \mathrm{g} /$ well) were transiently transfected into INS-1 cells using Lipofectamine-Plus reagent. After $6 \mathrm{~h}$ of transfection, media were replaced with normal culture media and incubated for an additional $24 \mathrm{~h}$. Transfected cells were serum-starved for $24 \mathrm{~h}$ and then stimulated with exendin- 4 for $18 \mathrm{~h}$. To control transfection efficiency, cells were cotransfected with $100 \mathrm{ng}$ of the internal control vector (pSV40-gal). Luciferase activities were normalised to $\beta$-galactosidase activity and data were expressed relative to the unstimulated value of the pCD1-827 construct.

\section{Chromatin immunoprecipitation assay}

Chromatin immunoprecipitation (ChIP) assays were performed with a chromatin immunoprecipitation assay kit (Upstate Biotechnology, Grand Island, NY, USA) according to the manufacturer's instructions. The conditions for PCR were as follows: $30 \mathrm{~s}$ at $95^{\circ} \mathrm{C}, 30 \mathrm{~s}$ at $55^{\circ} \mathrm{C}$ and $30 \mathrm{~s}$ at $72^{\circ} \mathrm{C}$ for 25 cycles, with final extension for $10 \mathrm{~min}$ at $72^{\circ} \mathrm{C}$. Primers for ChIP were as follows: forward, 5'CGGCGATTTGCATATCTACGAAGG-3'; reverse, 5'AAGCCGGGCAGAGAAAAAGGAG-3'.

Preparation of nuclear extracts and electrophoretic mobility shift assay

Preparation of nuclear proteins from INS-1 cells and electrophoretic mobility shift assays (EMSAs) was performed as described previously [15]. In supershift studies, $2 \mu \mathrm{g}$ of each antibody was preincubated with the nuclear extract for $10 \mathrm{~min}$ at $4^{\circ} \mathrm{C}$ before addition of the labelled probe. In competition experiments, the nuclear extract was incubated with a 100-fold molar excess of the appropriate unlabelled competitor oligonucleotides. Oligonucleotides used were as follows: oligo $3^{\prime}(-114$ to -87$)$, 5'TGCGCCCGCCTCGGCCCGCCCCCCCTCC-3'; oligo $5^{\prime}(-153$ to -134$), 5$ '-CCCACCCCCGCCGCTCACTG3'; oligo 5' EGR1 mutant, 5'-CCCACCCttGCCGCTCAC TG-3'; EGR1 consensus, 5'-GGATCCAGCGGGGGC GAGCGGGGGGCGA-3'; SP1 consensus, 5'-TGAAGCC CCGCCCCAACGGA-3'; and AP2 consensus, 5'-GATC GAACTGACCGCCCGCGGCCCGT-3'.
Statistical analysis

The relative band densities were quantified using Scion Imaging software (Scion Corporation, Frederick, MD, USA). Data were expressed as mean \pm SD. Statistical analysis was performed using one-way ANOVA and Student's $t$-test. Statistical significance was accepted at $p<0.05$.

\section{Results}

Effect of exendin-4 on Ccndl expression

INS-1 cells were serum-starved for $24 \mathrm{~h}$ and treated with exendin-4 (10 nmol/1). Total cellular RNA or protein was extracted at different periods. Exendin-4 significantly increased $C c n d 1$ expression. Ccndl mRNA level reached
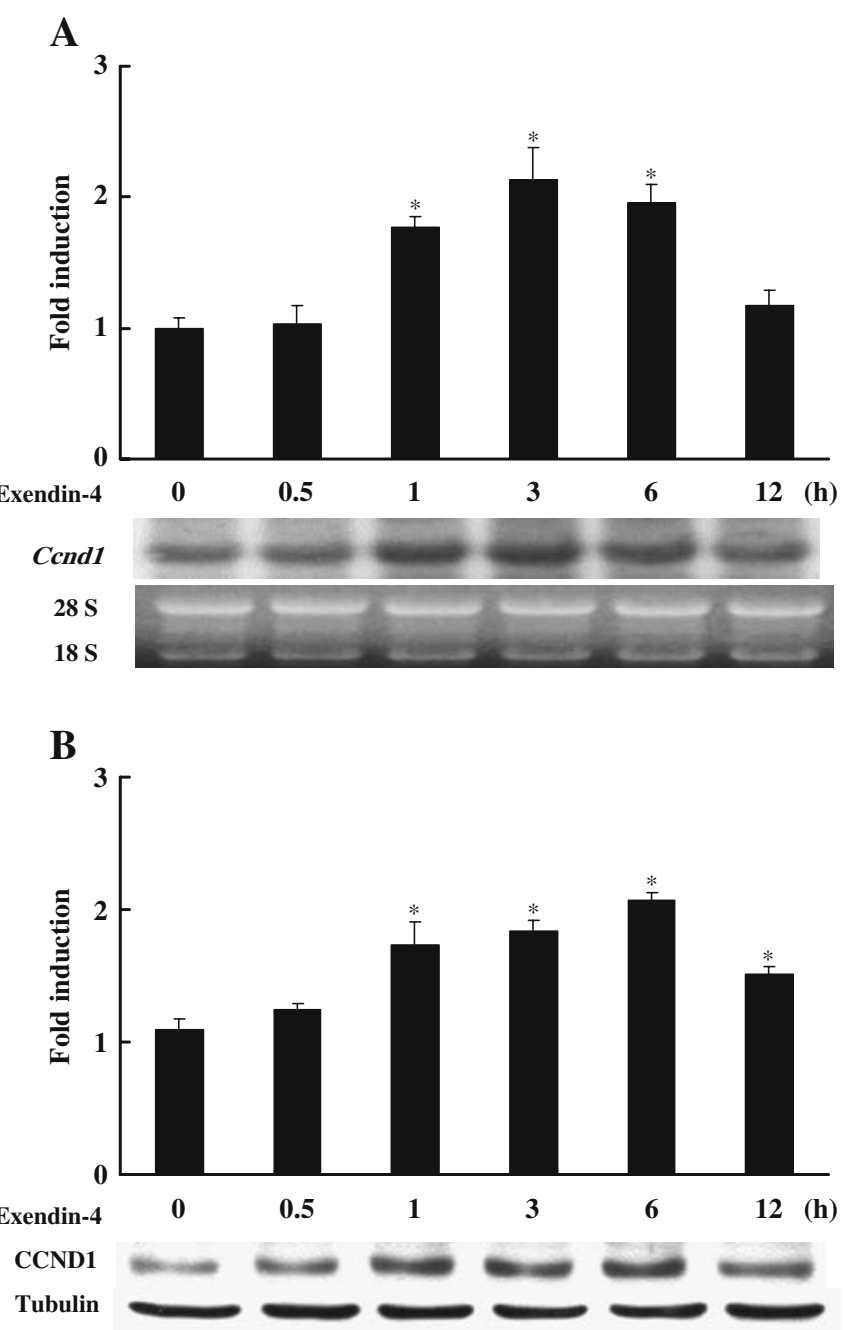

Fig. 1 Induction of $C c n d 1$ expression. a Northern blot analysis. Data are expressed as mean and SD density ratio of Ccnd 1 mRNA to $28 \mathrm{~S}$ RNA from three independent experiments. ${ }^{*} p<0.05$ vs unstimulated control. b Western blot analysis. Data are expressed as mean and SD density ratio of CCND1 to tubulin from three independent experiments. ${ }^{*} p<0.05$ vs unstimulated control 
its maximum at $3 \mathrm{~h}$ and returned to control level by $12 \mathrm{~h}$ (Fig. 1a). CCND1 protein level peaked at $6 \mathrm{~h}$ and had declined by $12 \mathrm{~h}$ (Fig. 1b).

Mapping the cis-regulatory elements responsible for exendin-4-induced transcriptional activation on the rat $C$ cnd 1 promoter

To evaluate the transcriptional activation of rat $C$ cnd 1 by exendin- 4 , the promoter activities were examined. Putative binding sites for trans-acting factors on the promoter are shown (Fig. 2a). Results of the transient transfection assay revealed that exendin- 4 increased the activities of the pCD1 $-827,-625,-446$ and -174 constructs approximately $2.6-$ fold. However, the stimulatory effect of exendin-4 was reduced to about 1.6-fold in pCD1 -73 and -34 (Fig. 2b), suggesting the location of the cis-regulatory elements, which are mainly involved in exendin-4-induced transcriptional activation of $C c n d 1$, between -174 and -73 .
Exendin-4 induction of specific interaction of nuclear protein with the region between -153 and -134 on the rat Ccndl promoter

The proximal region of rat Ccndl promoter contains several important cis-regulatory elements, such as $\mathrm{NF} \kappa \mathrm{B}$, SP1, CREB and two putative EGR1 binding sites (Fig. 3). Since the two EGR1 sites ( -151 to -143 and -108 to -100$)$ are located between the sequences of -174 and -73 , it is necessary to examine the involvement of these EGR1 binding sites of rat in exendin-4-induced Ccndl activation. The interaction of these EGR1 binding sites with nuclear transcriptional factors was tested by EMSAs. Two radioactively labelled synthetic oligonucleotide probes $(-153$ to -134 , oligo $5^{\prime}$ and -114 to -87 , oligo $3^{\prime}$ ) bearing each sequence of the putative EGR1 binding sites were prepared. Incubation of the oligo $3^{\prime}$ probe with nuclear extract of the INS-1 cells resulted in the binding of the oligonucleotide to multiple nuclear protein complexes. The binding activities were not influenced by exendin-4 treatment (Fig. 4a). However, when oligo $5^{\prime}$ was used as a probe, one protein complex was induced within $30 \mathrm{~min}$, continued until $1 \mathrm{~h}$, and then declined progressively up to $4 \mathrm{~h}$ (Fig. 4b, arrow), suggesting the involvement of the putative EGR1 binding site of -151 to -143 in the

A
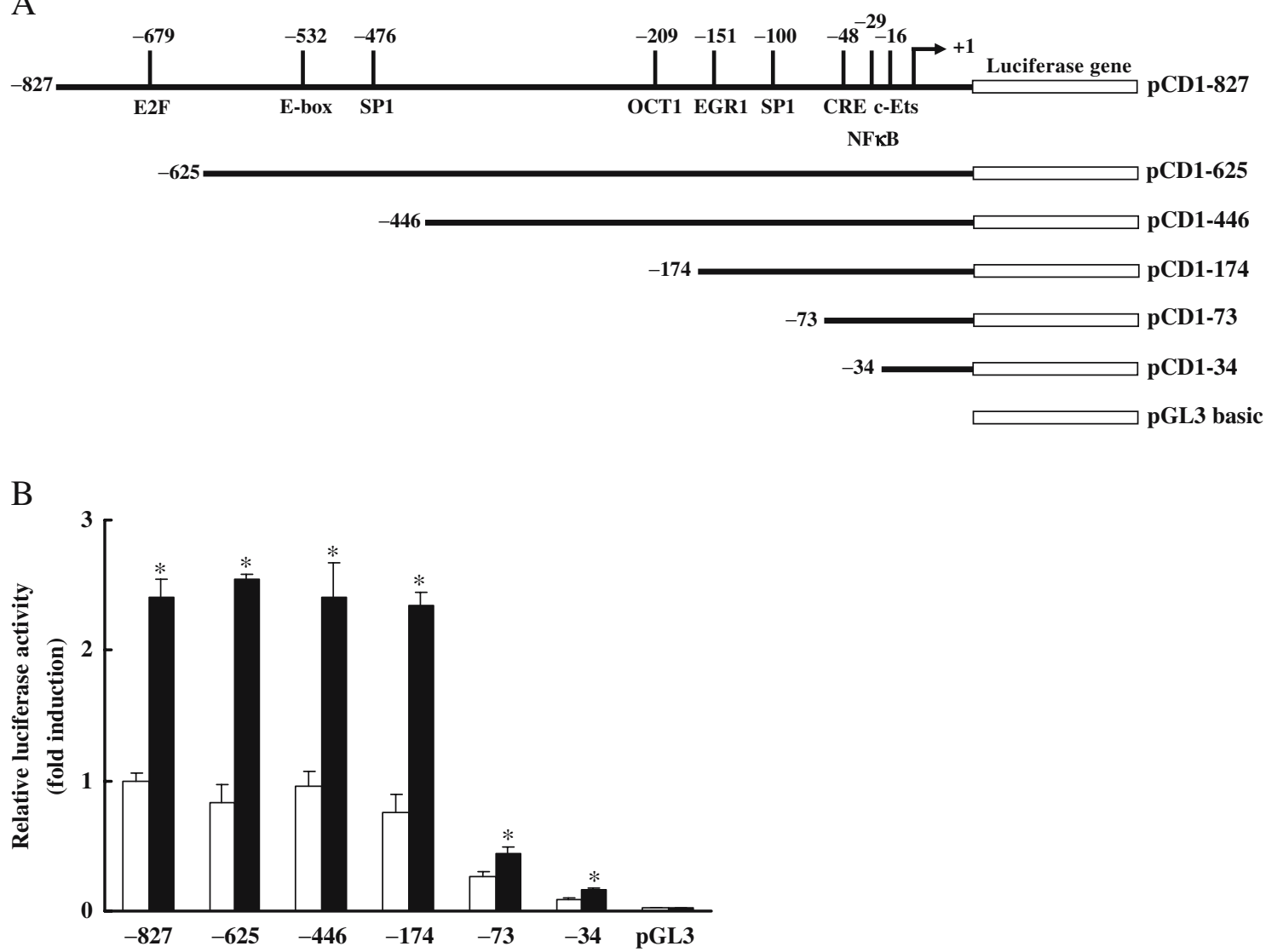

Fig. 2 The region responsible for induction of the rat Ccndl promoter. a Schematic diagram of the full-length and $5^{\prime}$ serially deleted promoter-luciferase constructs. Numbers at the top indicate the positions of the putative binding sites for regulatory factors starting from the transcription initiation site. b Promoter analysis of Ccndl deletion constructs. Data are mean and SD from three independent experiments. Filled bars, stimulated with exendin-4; open bars, unstimulated. ${ }^{*} p<0.05$ vs unstimulated self control 


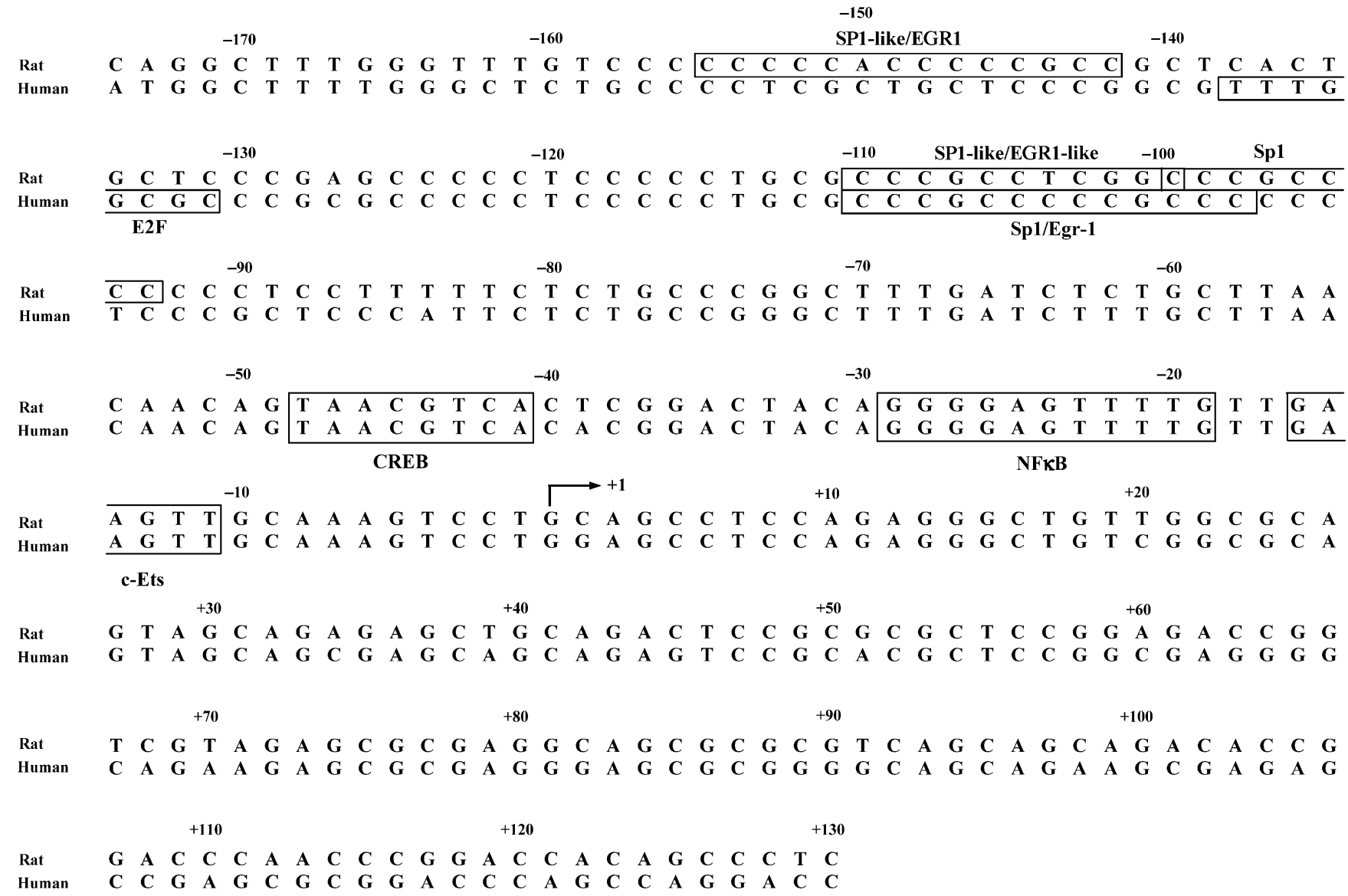

Fig. 3 Sequence comparison of the region from -174 to +130 in the rat Ccndl promoter with the corresponding sequence in human CCND1. The putative cis-regulatory elements are indicated by boxes. Alignment of the sequences was based on the transcription

interaction with nuclear protein to activate the Ccndl promoter in response to exendin-4.

Characterisation of the nuclear protein interacting with the region between -153 and -134 on the rat Ccndl promoter

To characterise the nuclear proteins that were bound to the oligo 5' probe, a supershift assay was performed. Because the oligo $5^{\prime}$ sequence is $\mathrm{G}+\mathrm{C}$-rich and contains putative EGR1 and SP1 binding sites, antibodies against EGR1 and SP1 were used. Antibody against AP2 transcription factor was used as control. Exendin-4-induced nuclear protein complex was completely abolished by preincubation with anti-EGR1 antibody but not with anti-SP1 or anti-AP2 antibodies (Fig. 5a). The competition assay also confirmed the binding specificity of EGR 1 to the oligo $5^{\prime}$ probe. As shown in Fig. 5b, a 100-fold molar excess of unlabelled oligo $5^{\prime}$ entirely competed away the binding activities of all complexes (lane 2). The exendin-4-induced complex was specifically competed away with a 100 -fold molar excess of unlabelled oligonucleotide bearing EGR1 consensus sequence. The oligonucleotides bearing a mutant oligo 5', consensus AP2 or SP1 sequence were without effect. Thus, start site and generated using the BioEdit program (version 7.0.5.2). The accession numbers of the genes are as follows: human, Z29078; rat, $\mathrm{AB} 042564$

it appears that the nuclear protein that was bound to the region between -153 and -134 of oligo $5^{\prime}$ in response to exendin-4 is EGR1.

Functional relevance of the EGR1 binding site in the rat Ccndl promoter activities

To determine whether the putative EGR1 site of the rat Ccndl described earlier was responsible for transducing the exendin- 4 signal, the putative EGR1 binding sequence $(-151$ CACCCCCGC -143) within the Cond1 promoter construct (pCD1 -827) was mutated to CACCCTTGC by site-directed mutagenesis. The wild-type or mutant EGR1 construct was transiently transfected into INS-1 cells and the promoter activity of each construct was analysed. As shown in Fig. 6, the mutant EGR1 construct showed a 34\% decrease in luciferase activity compared with the wild-type construct, indicating the involvement of an intact EGR1binding site on the proximal Ccndl promoter sequence in stimulating the expression of the rat Ccndl promoter by exendin- 4 . 
A

Oligo 3

-114 TGCGCCCGCCTCGGCCCGCCCCCCCTCC -87

$\begin{array}{lllllll}\text { Time (min): } & 0 & 15 & 30 & 60 & 120 & 240\end{array}$

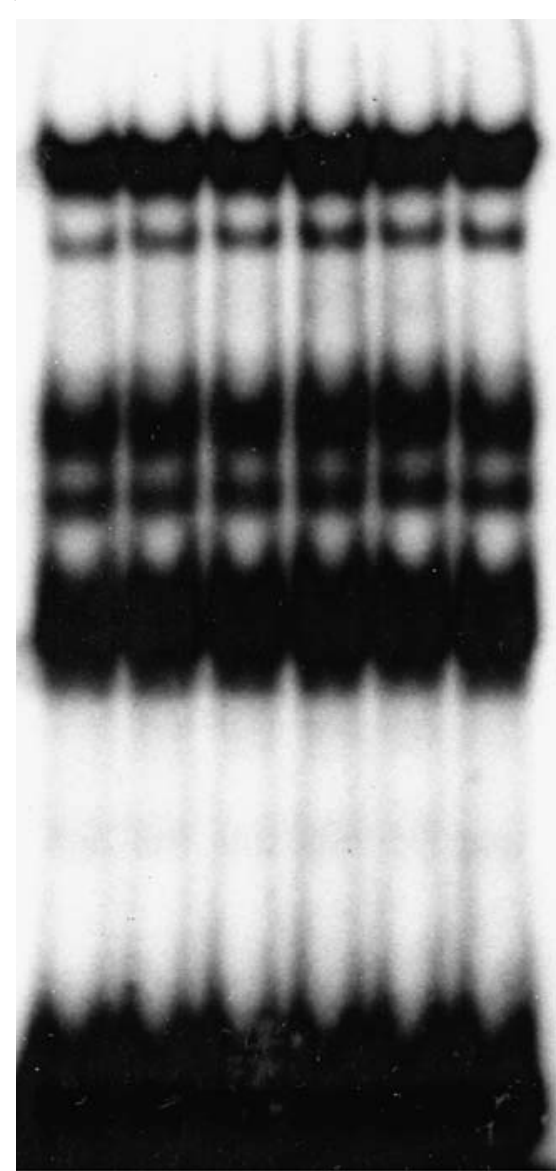

Fig. 4 Interaction of nuclear proteins with $5^{\prime}$ putative EGR1 binding site. Nuclear extracts were prepared after incubation of INS1 cells in the absence (zero time) or presence of exendin- $4(10 \mathrm{nmol} /$ 1) for the indicated time points. End-radiolabelled oligonucleotides

Recruitment of EGR1 to rat Ccnd1 promoter in vivo by exendin- 4

The effect of exendin- 4 in inducing the recruitment of EGR1 protein to the rat Ccnd1 promoter to activate the gene was investigated in vivo by ChIP assay. Purified DNAs were amplified by standard PCR using a pair of primers flanking the putative EGR1 binding region $(-213$ to -69 ) of the rat Ccndl promoter (Fig. 7a). As shown in Fig. $7 \mathrm{~b}$, in vivo binding of EGR 1 to the Ccndl promoter was clearly increased in response to exendin-4, while the binding of SP1 was not changed, indicating the recruitment of EGR1 to the chromatin around the exendin-4-responsive region.
B

Oligo 5'

-153 CCCACCCCCGCCGCTCACTG -134

$\begin{array}{lllllll}\text { Time (min): } & 0 & 15 & 30 & 60 & 120 & 240\end{array}$

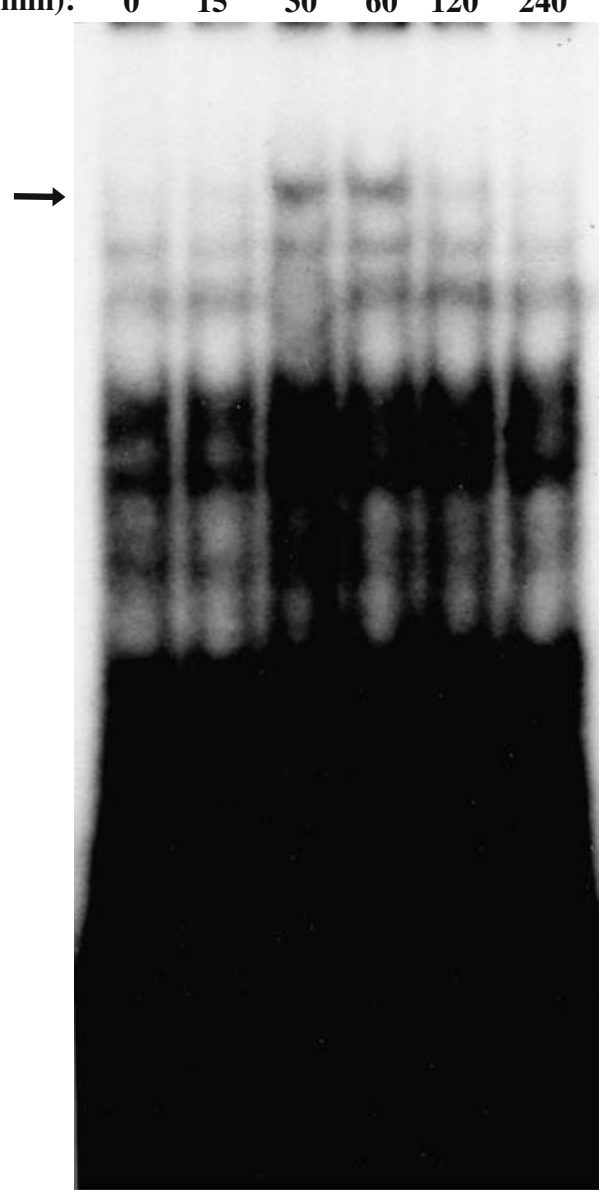

containing the $3^{\prime}$ (a) or $5^{\prime}$ (b) putative EGR1 binding site were incubated with the nuclear extracts. Experiments were repeated independently four times

Effect of exendin-4 on EGR1 production in INS-1 cells and primary pancreatic islets

We determined the kinetics of protein production. Production of EGR1 protein was induced maximally between 30 and $60 \mathrm{~min}$ and had declined rapidly by $240 \mathrm{~min}$ in response to exendin-4 (Fig. 8a). EGR1 is an immediateearly response protein that is newly synthesised upon stimulation $[19,20]$. Thus, for the interaction of EGR1 with the Ccnd1 promoter, de novo EGR1 protein synthesis is required. Serum-starved INS-1 cells pretreated with cycloheximide $(10 \mu \mathrm{g} / \mathrm{ml})$, a protein synthesis inhibitor, for $1 \mathrm{~h}$ and exposed to exendin- 4 for an additional $1 \mathrm{~h}$ failed to induce the endogenous EGR1 protein and to form the EGR1/DNA complex (Fig. 8b, arrow), suggesting the requirement of the newly synthesised EGR1 protein for the formation of the EGR1/DNA complex. Next, we examined the effect of newly synthesised transcription factors on the production of CCND1 protein. Pretreatment with cyclo- 
A

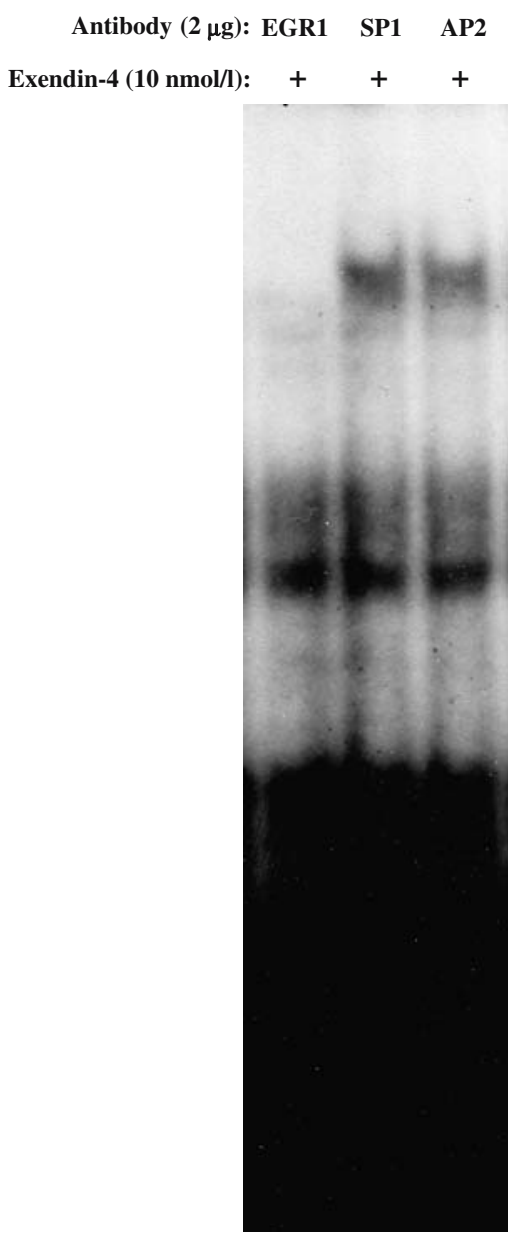

B

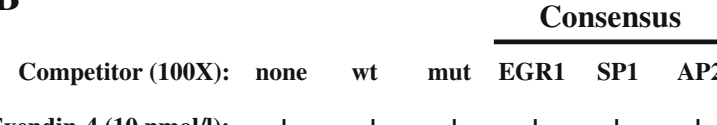

Exendin-4 $(10 \mathrm{nmol} / \mathrm{l}): \quad+\quad+\quad+\quad+\quad+\quad+\quad+$

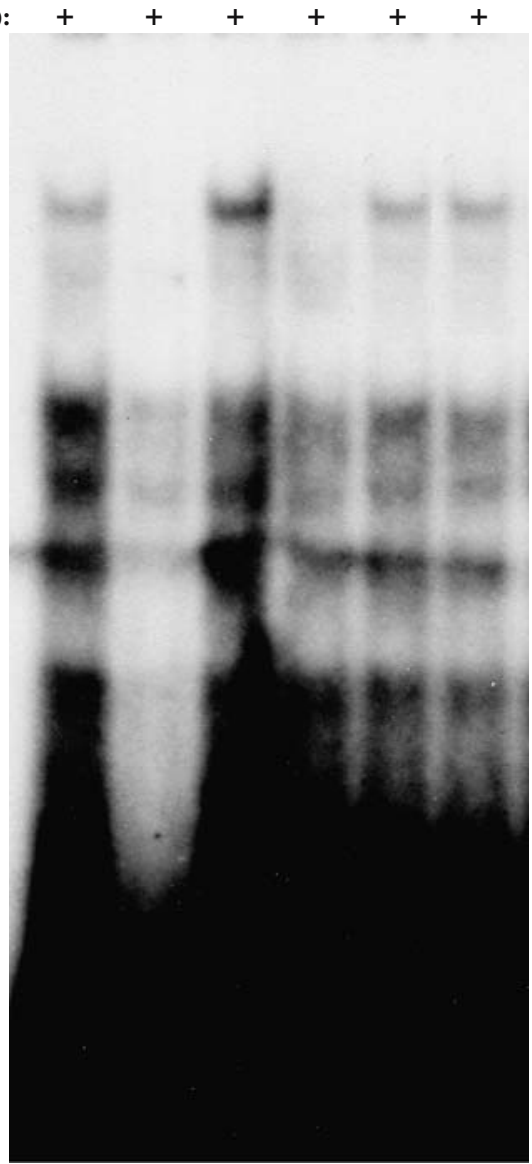

EGR1 wild

CCCACCCCCGCCGCTCACTG

CCCACCCttGCCGCTCACTG

EGR1 mutant

EGR1 consensus GGATCCAGCGGGGGCGAGCGGGGGGCGA

SP1 consensus TGAAGCCCCGCCCCAACGGA

AP2 consensus GATCGAACTGACCGCCCGCGGCCCGT

Fig. 5 Characterisation of the binding activity of 5' putative EGR1 binding site. a A supershift assay was performed with anti-EGR1, anti-SP1 and anti-AP2 antibodies. b A competition experiment was performed using a $100 \times$ molar excess of unlabelled oligonucleotides

heximide inhibited CCND1 production induced by exendin- 4 below the unstimulated level (Fig. 8c). The inductive response to exendin-4 also occurred in primary pancreatic islets. As shown in Fig. 8d, the islets significantly induced the EGR1 and CCND1 proteins.

\section{Discussion}

Exendin-4, a potential therapeutic agent in the treatment of type 2 diabetes, has been known as a growth factor in beta cells as well as in islets $[21,22]$. The molecular mechanisms governing the regulation of beta cell proliferation by exendin- 4 are not well understood. Therefore, the aim of this study was to investigate the transcriptional containing the wild-type, mutant EGR1 or three consensus EGR1, AP2 or SP1. Nuclear extracts were prepared from the exendin-4stimulated INS-1 cells for $1 \mathrm{~h}$. Experiments were repeated independently three times

regulation of $C$ cnd 1 by exendin- 4 in INS- 1 cells employing serial deletion constructs of rat $C$ cnd 1 promoter.

In this study, we found that exendin- 4 induced Ccnd1 mRNA and subsequent protein expression in INS-1 cells. The involvement of CCND1 protein in beta cell proliferation has recently been verified by others: overexpression of either $C c n d 1$ alone or in combination with $C d k 4$-induced beta cell proliferation in rat and human pancreatic islets [23], and CCND1 protein was shown to be essential for normal postnatal pancreatic beta cell growth [24]. These studies, together with our results, implicate CCND1 protein in beta cell proliferation, although CCND2 was also reported to trigger the progression of beta cell replication in the early postnatal period $[24,25]$. 


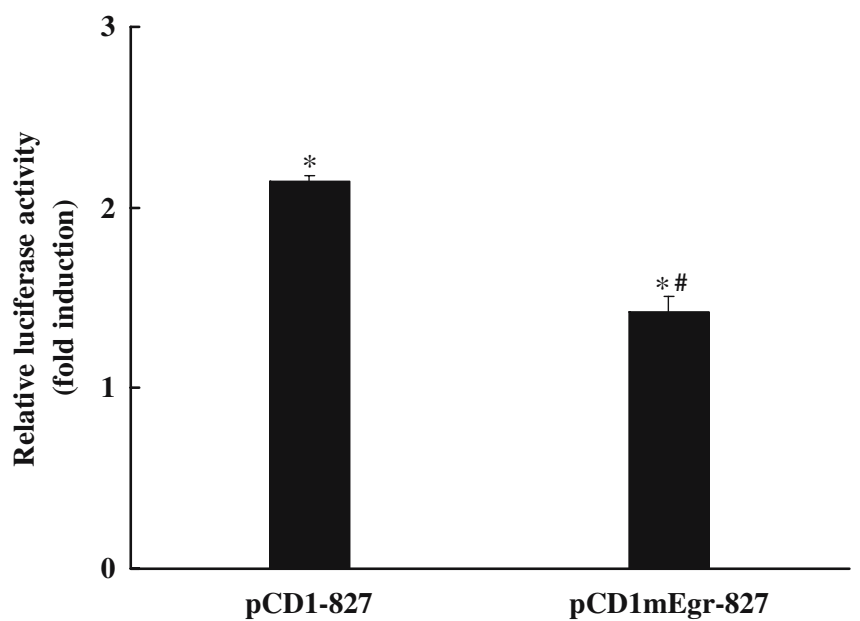

Fig. 6 Effect of mutation of the putative EGR1 site on the induction of the reporter gene. Luciferase activity induced by the wild-type (pCD1-827) and mutant EGR1 (pCD1mEgr-827) constructs. Data are mean and SD from five independent experiments. ${ }^{*} p<0.05$ vs unstimulated self-control. ${ }^{\#} p<0.05$ vs pCD1-827 construct

We clearly demonstrated that the induction of Ccnd1 expression by exendin- 4 occurred predominantly at the transcriptional level. Results of the promoter analysis revealed that the region between -174 and -73 was largely involved in Ccndl promoter activity, implicating this region as a major exendin-4-responsive element. The importance of this region has also been emphasised in human Ccnd1 promoters of oesophageal squamous carcinoma [26] and of Chinese hamster ovary cells stably overexpressing angiotensin II type 1A receptor [27]. Meanwhile, other cis-regulatory elements located between -73 and +130 of the promoter also appear to be involved in promoter activities in response to exendin-4. In fact, the CRE (cyclic AMP-responsive element) and c-Ets sites, located at -48 and -16 , respectively, have been demonstrated to be involved in induction of the transcriptional activity of human $C c n d 1$ [28, 29]. In particular, promotion of beta cell survival by GLP1 via the second messenger cyclic AMP was significantly reduced in mice deficient in CREB activity, leading to diabetes [30]. Thus, the increased activities of the -73 and -34 constructs in our promoter assay can be explained by the presence of these sites, which are conserved in both human and rat promoters (Fig. 3).

Our EMSAs demonstrated that the transcriptional activation of $C$ cndl was mediated by binding of EGR 1 to the
Fig. 7 Recruitment of EGR1 to the $C$ cnd 1 promoter in vivo. a The amplified region of rat Ccnd 1 promoter and primers used in the chromatin immunoprecipitation assay. b PCR was performed to amplify the region from -213 to -69 . The levels of binding of SP1 and EGR1 to the Ccndl promoter region were expressed relative to the band intensity of the unstimulated control. Input samples represent $0.05,0.01$ and $0.002 \%$ of the total DNA, whereas the PCRs of the immunoprecipitations include $5 \%$ of the resuspended DNA. Data are expressed as mean $( \pm \mathrm{SD})$ from three independent experiments. ${ }^{*} p<0.01$ vs unstimulated self control
A

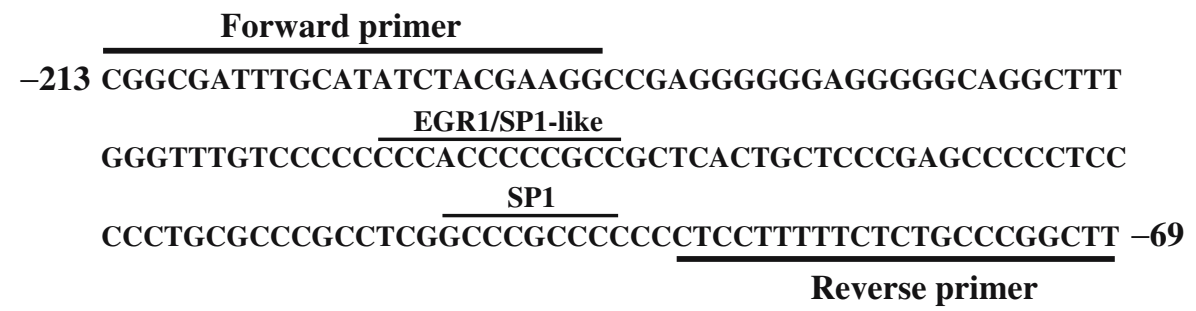

B
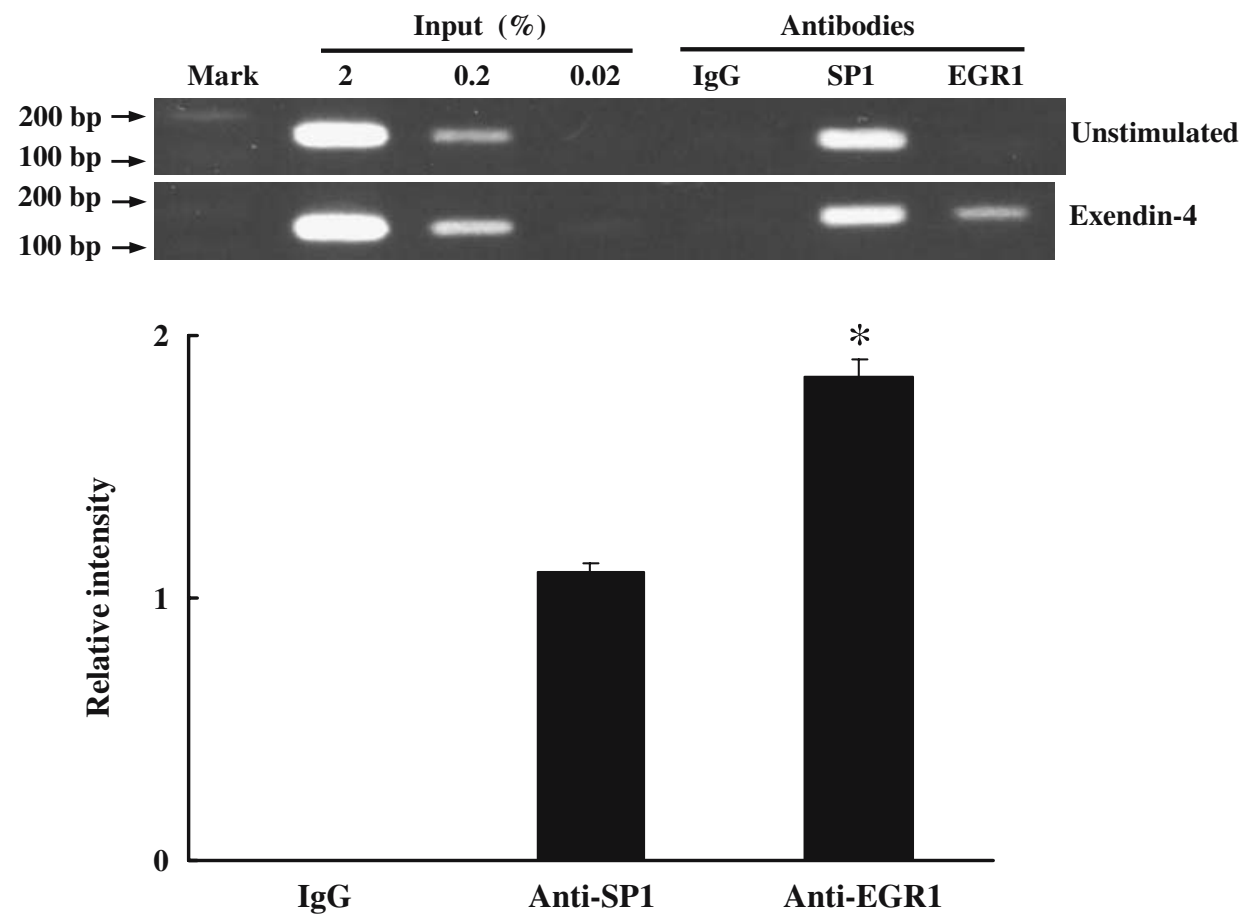
A

Exendin-4

$\mathbf{0}$

15

30

60

120

240

(min)

EGR1

Tubulin

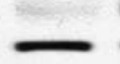

$\longrightarrow$

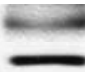

$+2$

$-$

B

Exendin-4 : $\quad-\quad+\quad+$

CHX $(10 \mu \mathrm{g} / \mathrm{ml}): \quad-\quad-\quad+$

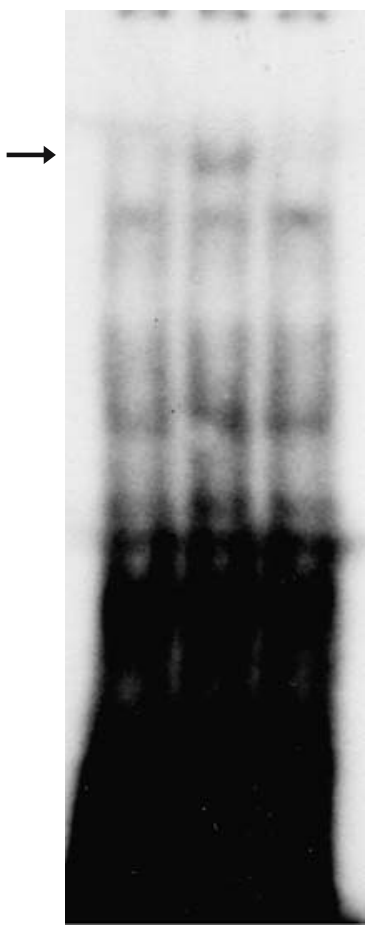

EGR1

Tubulin

C

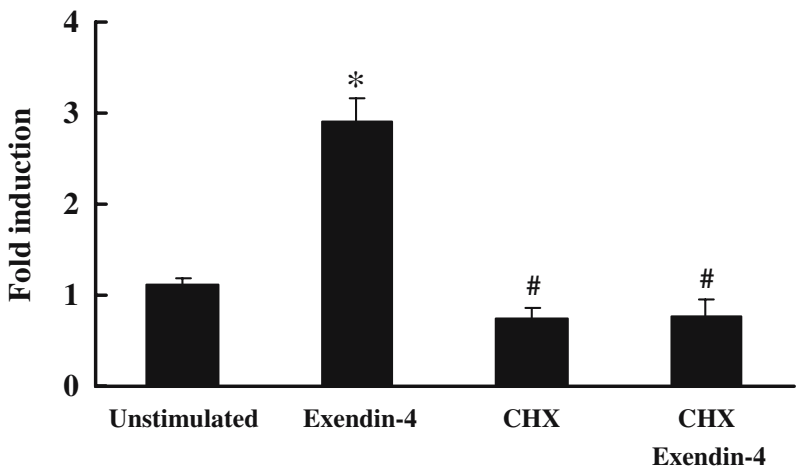

CCND1

Tubulin

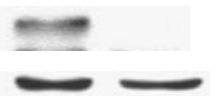

Fig. 8 Effects of exendin-4 on EGR1 expression in INS-1 cells and in isolated rat pancreatic islets. Western blot analysis (a) was performed to determine the time course of EGR1 expression. b Nuclear extracts were prepared and electrophoretic mobility shift assay $(E M S A)$ was performed with radiolabelled oligo $5^{\prime}$ probe containing the putative EGR1 binding site. Western blot analysis of EGR1 (below) was performed with the same samples used in EMSA. Experiments were repeated independently three times. c Inhibition of exendin-4 induction of CCND1 protein by cycloheximide $(\mathrm{CHX}) .{ }^{*} p<0.01$ vs unstimulated control, $\mathrm{CHX}$ or CHX plus exendin-4; ${ }^{*} p<0.05$ vs unstimulated control. d Induction of EGR1 and CCND1 proteins by exendin-4 in rat pancreatic islets. The islets were treated the same way as INS-1 cells. Data are mean and SD density ratio of CCND1 to tubulin from three independent experiments. ${ }^{*} p<0.05$ vs unstimulated control
D
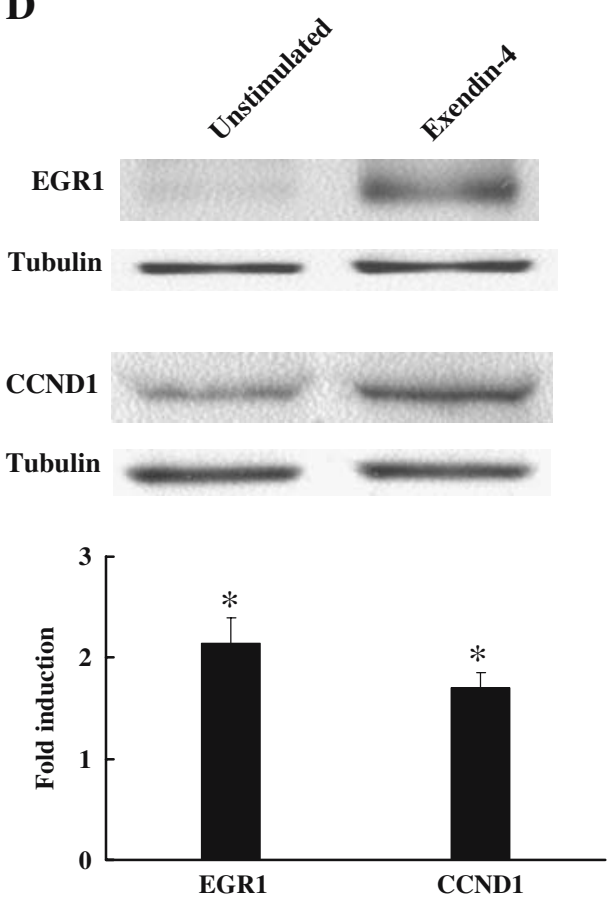
site $(-153$ to -134$)$ on rat $C c n d 1$ promoter. The antisense strand (5'-GCGGGGGTG-3') of the putative EGR1 sequence perfectly matches a consensus EGR1 binding sequence, 5'-GCG(T/G)(G/A)GG(C/A/T)G-3' [31]. Specific EGR1 binding to this putative binding site was confirmed by immune-supershift and competition assays. Moreover, we showed that the rat Ccndl promoter functionally interacted with EGR1 by site-directed mutation at the putative EGR1 site. These findings were confirmed by the ChIP assay, demonstrating a clear increase in EGR1 binding to the $C c n d 1$ promoter by exendin-4 in vivo.

However, the oligo $3^{\prime}$ probe that included another putative EGR1 binding site $(-108$ to -100$)$ failed to form a complex with EGR1. In humans, this EGR1 binding site, overlapping two SP1 binding sites, corresponds to the consensus EGR1 site and is essential for the binding of EGR1 in order to activate $C$ cnd 1 transcription in response to growth factors $[26,27]$. Comparing the sequences of the rat (5'-CGCCTCGGC-3') and human (5'-CGCCCCCGC$\left.3^{\prime}\right)$, the thymine and guanine at -104 and -102 in rats are replaced with cytosine in humans. Specifically, the cytosine residue at -102 has been described as a critical base for EGR1 binding [31]. Thus, the presence of a guanine residue at position -102 of the rat promoter may be unfavourable for EGR1 binding in our EMSA study.

The cis-regulatory sequence required for EGR1/SP1 binding is represented by the $\mathrm{G}+\mathrm{C}$-rich region of the promoter, in which EGR1 and SP1 binding sites generally overlap [26, 32, 33]. Therefore, a dual interplay between EGR1 and SP1 has been demonstrated for a number of gene promoters. In our EMSA using the oligo $5^{\prime}$ probe, which contains potential SP1 binding sites overlapping the EGR1 site (Fig. 3), SP1 binding activity was not observed in the supershift and competition assays (Fig. 5). However, SP1 was constitutively bound to the promoter irrespective of exendin- 4 treatment in the ChIP experiments. These results could have occurred because the amplified region contains consensus SP1 sequence (5'-CCCGCCC-3', -100 to -93). Meanwhile, the oligo $3^{\prime}$ probe containing the binding site of the consensus SP1 was supershifted by antiSP1 antibody but not by anti-EGR1 or anti-AP2 antibodies (data not shown). These results are consistent with previous studies showing binding of EGR1 to an overlapping EGR1/ SP1 site without displacement of SP1 in the human Ccnd1 promoter [26, 27].

The EGR1 transcription factor, also known as Krox24, Zif268 or Tis8, is a zinc-finger protein that is a critical upstream regulator of growth, proliferation and differentiation. Expression of Egrl is rapidly induced by mitogens, insulin and glucose in different cells, including beta cells $[19,20,34,35]$. In our experiments, the rapid and transient induction of EGR1 protein in response to exendin-4 (Fig. 8a) was consistent with previous studies $[9,34]$. In addition, the time course of EGR1 protein induction was consistent with that of EGR1 binding to the radiolabelled oligo $5^{\prime}$ probe (Fig. 4b). Moreover, the requirement for de novo EGR1 protein synthesis strongly supports the validity of our EMSA data in the experiment employing cyclohex- imide. The results of the study on the newly synthesised transcription factors using cycloheximide indicate that constitutively expressed transcription factors, as well as inducible transcription factors such as EGR1, may contribute to the production of CCND1 protein.

In summary, we found that exendin-4 rapidly induced the EGR1 transcription factor and that EGR1 was bound to its putative binding site between -151 and -143 on the rat Ccnd1 promoter, which is different from the functional EGR1 binding site (between -108 and -100 ) of humans. Through the EGR1-mediated induction of CCND1 protein, exendin-4 may contribute to beta cell proliferation.

Acknowledgement This study was supported by a grant from Korea Science and Engineering Foundation (R-2004-000-10127-0), Republic of Korea.

\section{References}

1. Deacon CF (2004) Therapeutic strategies based on glucagonlike peptide 1. Diabetes 53:2181-2189

2. Vilsboll T, Holst JJ (2004) Incretins, insulin secretion and type 2 diabetes mellitus. Diabetologia 47:357-366

3. Wang Q, Li L, Xu E, Wong V, Rhodes C, Brubaker PL (2004) Glucagon-like peptide-1 regulates proliferation and apoptosis via activation of protein kinase B in pancreatic INS-1 beta cells. Diabetologia 47:478-487

4. Stoffers DA, Kieffer TJ, Hussain MA et al (2000) Insulinotropic glucagon-like peptide 1 agonists stimulate expression of homeodomain protein IDX-1 and increase islet size in mouse pancreas. Diabetes 49:741-748

5. Buteau J, Foisy S, Joly E, Prentki M (2003) Glucagon-like peptide 1 induces pancreatic beta-cell proliferation via transactivation of the epidermal growth factor receptor. Diabetes 52:124-132

6. Buteau J, Roduit R, Susini S, Prentki M (1999) Glucagon-like peptide-1 promotes DNA synthesis, activates phosphatidylinositol 3-kinase and increases transcription factor pancreatic and duodenal homeobox gene 1 (PDX-1) DNA binding activity in beta (INS-1)-cells. Diabetologia 42:856-864

7. Susini S, Roche E, Prentki M, Schlegel W (1998) Glucose and glucoincretin peptides synergize to induce c-fos, c-Jun, junB, zif-268, and nur-77 gene expression in pancreatic beta(INS-1) cells. FASEB J 12:1173-1182

8. Schuppin GT, Pons S, Hugl S et al (1998) A specific increased expression of insulin receptor substrate 2 in pancreatic beta-cell lines is involved in mediating serum-stimulated beta-cell growth. Diabetes 47:1074-1085

9. Josefsen K, Sorensen LR, Buschard K, Birkenbach M (1999) Glucose induces early growth response gene (Egr-1) expression in pancreatic beta cells. Diabetologia 42:195-203

10. Montrose-Rafizadeh C, Avdonin P, Garant MJ et al (1999) Pancreatic glucagon-like peptide-1 receptor couples to multiple $\mathrm{G}$ proteins and activates mitogen-activated protein kinase pathways in Chinese hamster ovary cells. Endocrinology 140:1132-1140

11. Hunter T, Pines J (1994) Cyclins and cancer. II: Cyclin D and CDK inhibitors come of age. Cell 79:573-582

12. Sherr CJ (1994) G1 phase progression: cycling on cue. Cell 79:551-555

13. Hashemolhosseini S, Nagamine Y, Morley SJ, Desrivieres S, Mercep L, Ferrari S (1998) Rapamycin inhibition of the G1 to S transition is mediated by effects on cyclin D1 mRNA and protein stability. J Biol Chem 273:14424-14429 
14. Lin S, Wang W, Wilson GM et al (2000) Down-regulation of cyclin D1 expression by prostaglandin A (2) is mediated by enhanced cyclin D1 mRNA turnover. Mol Cell Biol 20: 7903-7913

15. Shiozawa T, Miyamoto T, Kashima H, Nakayama K, Nikaido T, Konishi I (2004) Estrogen-induced proliferation of normal endometrial glandular cells is initiated by transcriptional activation of cyclin D1 via binding of c-Jun to an AP-1 sequence. Oncogene 23:8603-8610

16. Matsumura I, Kitamura T, Wakao H et al (1999) Transcriptional regulation of the cyclin D1 promoter by STAT5: its involvement in cytokine-dependent growth of hematopoietic cells. EMBO J 18:1367-1377

17. Lee RJ, Albanese C, Stenger RJ et al (1999) pp60 (v-src) induction of cyclin D1 requires collaborative interactions between the extracellular signal-regulated kinase, p38, and Jun kinase pathways. A role for cAMP response elementbinding protein and activating transcription factor-2 in pp60 (v-src) signaling in breast cancer cells. J Biol Chem 274: $7341-7350$

18. Kim MJ, Ryu GR, Kang JH et al (2004) Inhibitory effects of epicatechin on interleukin-1beta-induced inducible nitric oxide synthase expression in RINm5F cells and rat pancreatic islets by down-regulation of NF-kappaB activation. Biochem Pharmacol 68:1775-1785

19. Christy BA, Lau LF, Nathans D (1988) A gene activated in mouse $3 \mathrm{~T} 3$ cells by serum growth factors encodes a protein with 'zinc finger' sequences. Proc Natl Acad Sci USA 85:7857-7861

20. Sukhatme VP, Cao XM, Chang LC et al (1988) A zinc fingerencoding gene coregulated with c-fos during growth and differentiation, and after cellular depolarization. Cell 53:37-43

21. Gedulin BR, Nikoulina SE, Smith PA et al (2005) Exenatide (exendin-4) improves insulin sensitivity and \{beta\}-cell mass in insulin-resistant obese fa/fa Zucker rats independent of glycemia and body weight. Endocrinology 146:2069-2076

22. Xu G, Stoffers DA, Habener JF, Bonner-Weir S (1999) Exendin-4 stimulates both beta-cell replication and neogenesis, resulting in increased beta-cell mass and improved glucose tolerance in diabetic rats. Diabetes 48:2270-2276

23. Cozar-Castellano I, Takane KK, Bottino R, Balamurugan AN, Stewart AF (2004) Induction of beta-cell proliferation and retinoblastoma protein phosphorylation in rat and human islets using adenovirus-mediated transfer of cyclin-dependent kinase4 and cyclin D1. Diabetes 53:149-159
24. Kushner JA, Ciemerych MA, Sicinska E et al (2005) Cyclins D2 and D1 are essential for postnatal pancreatic beta-cell growth. Mol Cell Biol 25:3752-3762

25. Georgia S, Bhushan A (2004) Beta cell replication is the primary mechanism for maintaining postnatal beta cell mass. J Clin Invest 114:963-968

26. Yan YX, Nakagawa H, Lee MH, Rustgi AK (1997) Transforming growth factor-alpha enhances cyclin D1 transcription through the binding of early growth response protein to a cis-regulatory element in the cyclin D1 promoter. J Biol Chem 272:33181-33190

27. Guillemot L, Levy A, Raymondjean M, Rothhut B (2001) Angiotensin II-induced transcriptional activation of the cyclin D1 gene is mediated by Egr-1 in CHO-AT (1A) cells. J Biol Chem 276:39394-39403

28. Nagata D, Suzuki E, Nishimatsu H et al (2001) Transcriptional activation of the cyclin D1 gene is mediated by multiple ciselements, including SP1 sites and a cAMP-responsive element in vascular endothelial cells. J Biol Chem 276:662-669

29. Albanese C, Johnson J, Watanabe G et al (1995) Transforming p21ras mutants and c-Ets-2 activate the cyclin D1 promoter through distinguishable regions. J Biol Chem 270:2358923597

30. Jhala US, Canettier G, Screaton RA et al (2003) cAMP promotes pancreatic beta-cell survival via CREB-mediated induction of IRS2. Genes Dev 17:1575-1580

31. Swirnoff AH, Milbrandt J (1995) DNA-binding specificity of NGFI-A and related zinc finger transcription factors. Mol Cell Biol 15:2275-2287

32. Bahouth SW, Beauchamp MJ, Vu KN (2002) Reciprocal regulation of beta(1)-adrenergic receptor gene transcription by $\mathrm{Sp} 1$ and early growth response gene 1: induction of EGR-1 inhibits the expression of the beta(1)-adrenergic receptor gene. Mol Pharmacol 61:379-390

33. Khachigian LM, Williams AJ, Collins T (1995) Interplay of Sp1 and Egr-1 in the proximal platelet-derived growth factor Achain promoter in cultured vascular endothelial cells. J Biol Chem 270:27679-27686

34. Keeton AB, Bortoff KD, Bennett WL, Franklin JL, Venable DY, Messina JL (2003) Insulin-regulated expression of Egr-1 and Krox20: dependence on ERK1/2 and interaction with p38 and PI3-kinase pathways. Endocrinology 144:5402-5410

35. Silverman ES, Collins T (1999) Pathways of Egr-1-mediated gene transcription in vascular biology. Am J Pathol 154: $665-670$ 